\title{
The beneficial roles of insulin and parathyroid hormones in the treatment of experimentally induced diabetic osteoporosis in female rats: bone mineral density, morphometric and histological studies
}

\author{
G.S. Abd El Aziz¹, W.S. Ramadan¹, M.O. El-Fark1, 2, H.A.M. Saleh¹ \\ ${ }^{1}$ Department of Anatomy, Faculty of Medicine, King Abdulaziz University, Jeddah, Saudi Arabia \\ ${ }^{2}$ Department of Anatomy, Faculty of Medicine, Suez Canal University, Egypt
}

[Received: 7 October 2015; Accepted: 10 November 2015]

\begin{abstract}
Background: Diabetes mellitus (DM) and osteoporosis are two frequent medical conditions with an increasing prevalence in elderly people and are responsible for large number of incurable fractures. This study is designed experimentally in female rats in order to determine whether combined treatment of insulin and parathyroid hormone (PTH) enhances the reversibility of the osteoporotic changes that occurred in streptozotocin (STZ)-induced DM.

Materials and methods: In this study, 30 adult female rats aged 3 months were used, they were randomly divided into: control group (6 rats) and diabetes group (24 rats), in which experimental DM was induced by i.p. injection of a single dose of STZ (60 mg/kg/body weight). Diabetic group was further divided into four subgroups (6 rats each): non-treated diabetic, insulin-treated (8-12 units s.c./day of Humalin U-40), PTH-treated (6.0 $\mu \mathrm{g}$ s.c. $/ \mathrm{kg} /$ day) and combined insulin and PTH-treated subgroups. All tested groups were assessed for body weight, food and water consumptions.

Results: At the end of the experimental period, the bone mineral density (BMD) was measured for all rats of different groups; then the rats were sacrificed and blood samples were collected for measuring glucose, alkaline phosphatase and osteocalcin levels. Right femora were dissected out and subjected to measurement of diameter of neck and shaft, length of shaft, and weight. Then the femora specimens were processed and stained with haematoxylin and eosin for histological study. The results showed that there was a statistically significant, decrease in $B M D$, increase in the level of alkaline phosphate, and decrease in the level of osteocalcin in rats in diabetic group compared with other groups; these parameters improved in other groups, especially in diabetes/insulin/PTH group. The rats in diabetic group showed statistically significant decrease in neck and shaft diameters and weight of femur bone compared with other groups, while rats in diabetes/insulin/PTH group showed a significant improvement of these parameters. In diabetic group, there were different histopathological changes in cortical bone and Haversian canals, which improved in other groups, especially in rats in diabetes/insulin/PTH group.

Conclusions: The untreated DM resulted in dramatic reduction in BMD and morphometric parameters. Treatment with insulin ameliorated these effects to some extent, while PTH co-treatment had a more positive effect. The combination of PTH and insulin resulted in stronger improvement of all parameters to approximately like those of control rats. (Folia Morphol 2016; 75, 3: 341-354)
\end{abstract}

Key words: diabetes mellitus, osteoporosis, insulin, parathormone

Address for correspondence: M.M.O. El-Fark, MSc, MD, Assistant Professor of Anatomy and Embryology, College of Medicine, King Abdul Aziz University (KAU), Jeddah, P.O. Box: 80205 Jeddah 21589, Saudi Arabia, e-mail: anatomist1996@yahoo.com 


\section{INTRODUCTION}

Osteoporosis is a main bone complication in patients with diabetes mellitus (DM) $[9,25]$. Clinically speaking, it is well established that osteoporosis and DM are prevalent diseases with significant association of morbidity and mortality that represent very imperative public health concerns. Patients with DM have an increased risk of bone fractures [26]. Recognition of at-risk patients is critical in both prevention and treatment of osteoporosis. All diabetes-related factors should be considered in assessing osteoporosis and fracture risk reduction should be recommended to diabetic patients. Many agents are available for the prevention and treatment of osteoporosis in the diabetic population [26].

Diabetes mellitus is a pandemic debilitating metabolic disease that poses serious problems to health, community resources and socioeconomic services due to its dramatic increase $[3,48]$. The increased incidence of DM could be attributed to longer life expectancy associated with minimal physical activity and increased intake of high energy food [22, 47]. The World Health Organisation report in 2009 estimated that more than 180 million people with DM are present worldwide, and are expected to be more than double by 2030 [60, 61].

Diabetes mellitus is a complex disorder producing functional impairment in multiple organ systems $[3,48]$. Besides to its well-known complications such as retinopathy, nephropathy, neuropathy and arteriosclerosis, it could cause also many bone complications $[10,11,32]$. It is well recognised that the diabetic patients have various skeletal disorders, including decreased bone density (osteopoenia), which might be due to a deficit in bone formation [33, 34], decreased bone volume [6], alterations in calcium, phosphate and bone metabolism [46], increased fracture rates $[28,48]$, and delayed fracture healing [42].

Understanding the mechanism of diabetic bone loss is essential to determine the best therapy. In the majority of cases, osteoporosis in DM is treated the same as osteoporosis in subjects without diabetes [37]. Osteoporotic medications are mainly divided into two categories: antiresorptive medications and bone-forming (anabolic) medications [57]. Antiresorptive drugs e.g. bisphosphonates (alendronate, ibandronate and risedronate) are clinically used to treat osteoporotic spine and hip fractures [10]. Bisphosphonates are indicated for prevention as well as treatment of osteoporosis in diabetic postmenopau- sal women [24]. However, some reports suggested that DM may be a risk factor for bisphosphonaterelated osteonecrosis and therefore, that patients with DM being treated with bisphosphonates should be carefully monitored [31].

Currently, the only anabolic treatment approved for use in developed countries is the parathyroid hormone (PTH) [59]. Intermittent administration of PTH has been shown to have a potent anabolic effect on bone in various animal models and humans [2]. PTH can increase bone mass in osteoporotic humans and rats with osteopoenia induced by various causes [30, $55,56]$. Several studies have reported that intermittent administration of human PTH also enhances fracture healing of cortical bones such as shaft of femur or tibia after surgical intervention in animals [4, 41], even in an osteoporotic animal model [27]. Therefore, PTH is the only one candidate for the treatment of diabetesinduced osteoporosis [55].

Furthermore, it was reported that insulin has an anabolic effect on cartilage and bone [53], regardless of whether the primary effect of insulin is to reverse hyperglycaemia or whether there are direct effects of insulin on cells [7, 15]. Also, other studies have shown that insulin treatment normalised the cellular proliferation, chondrogenesis, mineralisation and mechanical strength of diabetic fracture healing $[7,16]$.

Therefore, it was expected that the combination of insulin and PTH would be more effective than each alone in the treatment of diabetic osteoporosis. Consequently, the aim of this study was to evaluate the beneficial effect of combined insulin and PTH treatment on the recovery of the osteoporotic changes that occurred in streptozotocin (STZ)-induced diabetes in rats.

\section{MATERIALS AND METHODS}

\section{Experimental design and animal grouping}

Thirty adult female Sprague-Dawley rats were used in this study. The rats were 3-month-old and body weight ranged 200-250 g. They were purchased from the animal house of King Fahd Research Centre and were housed in large plastic cages under the normal laboratory conditions, environmentally controlled $\left(25^{\circ}\right.$, 12-h light/12-h dark cycles). The rats were fed a commercial standard diet. Food and water were freely available. All experimental protocols were approved by the Unit of Biomedical Ethics Research Committee, Faculty of Medicine, King Abdulaziz University [(HA-02-J-008) No. of registration at National Committee of Bio. \& Med. Ethics]. In addition, the animals were adapted to 
the environment for 1 week before starting the experiment and then they were randomly divided into the following groups.

Control group (6 rats) - in which the rats were injected intraperitoneally with normal saline $(0.9 \%$ $\mathrm{NaCl}$ solution) in equivalent amounts to that of the treated groups.

Diabetic group (24 rats) - in which the rats were rendered diabetic. DM was induced by an intraperitoneal (i.p.) injection of a single dose of STZ ( $60 \mathrm{mg} / \mathrm{kg}$ body weight) after $18 \mathrm{~h}$ fasting [35]. STZ was dissolved in $0.1 \mathrm{M}$ citrate buffer ( $\mathrm{pH} 4.5$ ) immediately before use. Seventy-two hours following the injection, tail blood samples was obtained for measuring blood glucose concentration by using glucometer (SmartDC, Germany). Animals were classified as diabetics when glycaemia exceed $300 \mathrm{mg} / \mathrm{dL}$ [51]. All hormonal treatments were started 2 months after induction of DM by STZ injection, and then the rats were divided into four subgroups ( 6 rats each) as follows:

- Diabetes group - in which the diabetic rats were left without any treatment;

- Diabetes/insulin group - in which the diabetic rats were given insulin subcutaneously (Humalin U-40) at a dose of 8-12 units per day, daily for 2 months. The dose of insulin was adjusted to blood glucose level every 2 days [51];

- Diabetes/PTH group - in which the diabetic rats were given PTH (dissolved in $0.1 \mathrm{M}$ sodium acetate buffer, $\mathrm{pH}$ 7.2) subcutaneously at a dose $6.0 \mu \mathrm{g} / \mathrm{kg}$ per day for 2 months. The dose of PTH was based on previous studies, which showed that treatment with low-dose PTH $(6.0 \mu \mathrm{g} / \mathrm{kg} /$ day $)$ exerted anabolic effects on bone formation and bone mass [55];

- Diabetes/insulin/PTH group - in which the diabetic rats were given PTH and insulin together in the same doses as above.

Both food $(\mathrm{g})$ and water $(\mathrm{mL})$ consumptions of rats were measured daily. Body weight $(\mathrm{g})$ of the rats was measured at the beginning of the study (initial body weight) and at the end of the study (final body weight), and then the body weight difference (final-initial weight) was calculated.

\section{Drugs and hormones}

All chemicals, drugs and hormones used in this study were purchased through local scientific agents, Jeddah, KSA. STZ [(N-(methylonitrosocarbamoilo) Dglucosamine) (Sigma, Chemical Company, St. Louis,
USA)], insulin hormone [Insulin solution, Sigma, Chemical Company, St. Louis, USA] and parathyroid hormone fragment 1-34 human [Sigma, Chemical Company, St. Louis, USA].

\section{Methods}

At the end of the experiment, the following techniques were adopted:

Bone mineral density (BMD). BMD was measured in both femur and tibia (right and left sides) for all rats of the different experimental groups under anaesthetisation with i.p. injection of $4 \mathrm{~mL}$ of mixture $3: 1$ (ketamine $3 \mathrm{mg} / \mathrm{kg}$ and seton $1 \mathrm{mg} / \mathrm{kg}$ ) [39]. The measurement was done by dual-energy $\mathrm{X}$-ray absorptiometry (DEXA) using a LUNAR Prodigy Model, SA1002XR01, General electric, Madison, WI, USA, in the Centre of Excellence for Osteoporosis Research (CEOR), KAU, Jeddah, KSA adapted for measurements of small animals.

Biochemical analysis. The rats were sacrificed, blood was collected by cardiac puncture and blood samples were centrifuged at $3000 \mathrm{rpm}$ for $15 \mathrm{~min}$. The samples were divided into aliquots; plasma on sodium fluoride for glucose assessment which would be measured immediately and sera were used for all other parameters (serum alkaline phosphatase and osteocalcin); all parameters were measured by the commercially available kits. Sera were stored at $-80^{\circ} \mathrm{C}$ until assayed.

Bone morphometric study. The femora of rats of different groups were dissected out from the sacrificed animals. The following morphometric measurements were taken: diameter of the median points of the femur neck, diameter of the shaft of the femur, length of the shaft of the femur, and femur weight. All the measurements were obtained by the same experimenter, using a millimetre metallic ruler and a "Vernier Calibre". The measurements represented the arithmetic mean of three repetitions for each parameter analysed.

Bone histopathological procedures. The femora were cut distally, $5 \mathrm{~mm}$ proximally to the medial condyle articular surface. The cut specimens were placed in neutral buffered formalin solution for $48 \mathrm{~h}$, demineralised in $7.5 \%$ ethylene diaminetetraacetic acid (EDTA) in $0.1 \mathrm{~mol} / \mathrm{L}$ cacodylate buffer over 1 week, rinsed in buffer, and then stored in $70 \%$ ethanol. They were then dehydrated through a graded series of ethanol and acetone and embedded in paraffin wax. $5 \mu$ m thick frontal sections were cut from femurs, using a rotatory 
microtome (Leica pharmaceutical company, Germany), and mounted on glass slides. Sections were stained with haematoxylin and eosin for routine histological study.

\section{Statistical analysis}

Statistical analyses were performed by using SPSS program version 16 (IBM-USA). One way-analysis of variance (ANOVA) with Bonferroni post hoc test was carried out. Data were presented as mean \pm standard deviation (SD). $\mathrm{P}<0.05$ was considered statistically significant.

\section{RESULTS}

Body weight changes, food and water consumption

As shown in Table 1, there was a considerable increase in body weight after 2 months in control group $(21.29 \%)$, while rats in both diabetes and diabetes/PTH groups showed a statistically significant reduction of body weight, compared with the other study groups, at the end of the study (6\% in diabetes group and $5.76 \%$ in diabetes/PTH group). However, administration of insulin resulted in an increase in body weight gain in rats in both diabetes/insulin and diabetes/insulin/PTH treated groups (9\% and $8.8 \%$, respectively), with a statistically significant reduction compared with the control group. Also, food and water consumptions were increased in rats in both diabetes and diabetes/PTH groups with a statistically significantly difference when compared with the other study groups; however, administration of insulin in both diabetes/insulin, and diabetes/insulin/PTH re- sulted in a reduction of food and water consumption to be approximated to that in control group.

\section{Bone mineral density}

The BMD of the lower limbs was the highest in control group, followed by diabetes/insulin/PTH, diabetes/PTH, diabetes/insulin groups, and finally it was the lowest in diabetes group. There was a statistically significant decrease in BMD in rats in diabetes group when compared with control diabetes/PTH and diabetes/insulin/PTH groups. Rats from diabetes/PTH group showed a statistically significant decrease in BMD when compared with controls. Also, rats from diabetes/insulin group showed a statistically significant decrease in BMD when compared with both control and diabetes/insulin/PTH groups (Table 2).

\section{Biochemical results}

Diabetic rats in both diabetes and diabetes/PTH treated groups showed a statistically significant marked hyperglycaemia when compared with the control, diabetes/insulin, and diabetes/insulin/PTH treated groups (Table 3).

Regarding the level of serum alkaline phosphatase, it was in the normal range in the control group, while it was elevated in diabetes group and it was elevated to some extent in the other groups (diabetes/insulin, diabetes/PTH and diabetes/insulin/PTH). There was a statistically significant increase in the level of alkaline phosphate in rats in diabetic group when compared

Table 1. Body weight changes, food and water consumption

\begin{tabular}{lccccc}
\hline Parameters & Control & Diabetes & Diabetes/insulin & Diabetes/PTH & $\begin{array}{c}\text { Diabetes/insulin/ } \\
\text { /PTH }\end{array}$ \\
\hline Initial body weight [g] & $245.2 \pm 4.5$ & $247.4 \pm 3.7$ & $243.2 \pm 4.8$ & $244.7 \pm 3.8$ & $242.8 \pm 4.2$ \\
Final body weight [g] & $297.4 \pm 8.9$ & $232.5 \pm 4.2^{\mathrm{c}, \mathrm{g}, \mathrm{h}}$ & $265.1 \pm 7.6^{\mathrm{b}}$ & $230.6 \pm 4.6^{\mathrm{c}, \mathrm{g}, \mathrm{h}}$ & $264.3 \pm 6.9^{\mathrm{b}}$ \\
Body weight difference & $52.2 \pm 4.4$ & $-14.9 \pm 0.9^{\mathrm{d}}$ & $21.9 \pm 2.8^{\mathrm{c}}$ & $-14.1 \pm 0.8^{\mathrm{d}}$ & $21.5 \pm 2.7^{\mathrm{c}}$ \\
Food consumption [g/day] & $24.5 \pm 5.2$ & $42.8^{\mathrm{c}} \pm 9.6^{\mathrm{a}}$ & $29.8 \pm 6.7$ & $42.3 \pm 9.8^{\mathrm{a}}$ & $30.2 \pm 7.1$ \\
Water consumption [mL/day] & $30.2 \pm 7.1$ & $89.4 \pm 12.6^{\mathrm{c}, \mathrm{e}, \mathrm{f}}$ & $39.6 \pm 9.3$ & $90.1 \pm 11.9^{\mathrm{c}, \mathrm{e}, \mathrm{f}}$ & $39.9 \pm 9.8^{\mathrm{m}}$ \\
\hline
\end{tabular}

Results are expressed as mean \pm standard deviation; $n=6$. Significance of differences between groups was evaluated by one-way ANOVA followed by Bonferroni post-hoc test; $a-p<0.05$ vs. control group; $b-p<0.01$ vs. control group; $c-p<0.001$ vs. control group; $d-p<0.0001$ vs. control group; $e-p<0.01$ vs. diabetes/insulin group; $\mathrm{f}-\mathrm{p}<0.01$ vs. diabetes/insulin/PTH group; $\mathrm{g}-\mathrm{p}<0.0001$ vs. diabetes/insulin group; $\mathrm{h}-\mathrm{p}<0.0001$ vs. diabetes/insulin/PTH group

Table 2. Bone mineral density (BMD)

\begin{tabular}{|c|c|c|c|c|c|}
\hline Parameters & Control & Diabetes & Diabetes/insulin & Diabetes/PTH & $\begin{array}{c}\text { Diabetes/insulin/ } \\
\text { /PTH }\end{array}$ \\
\hline $\mathrm{BMD}\left[\mathrm{mg} / \mathrm{cm}^{2}\right]$ & $0.086 \pm 0.004$ & $0.074 \pm 0.004^{b, d, e}$ & $0.078 \pm 0.004^{b, c}$ & $0.081 \pm 0.003^{a}$ & $0.084 \pm 0.004$ \\
\hline
\end{tabular}


Table 3. Biochemical parameters

\begin{tabular}{|c|c|c|c|c|c|}
\hline Parameters & Control & Diabetes & Diabetes/insulin & Diabetes/PTH & $\begin{array}{c}\text { Diabetes/insulin/ } \\
\text { /PTH }\end{array}$ \\
\hline Glucose [mg/dL] & $122.4 \pm 21.9$ & $485.9 \pm 44.5^{b, d, g}$ & $131.4 \pm 24.5$ & $483.6 \pm 45.8^{b, d, g}$ & $132.7 \pm 26.3$ \\
\hline $\begin{array}{l}\text { Serum alkaline phosphatase } \\
{[\mathrm{U} / \mathrm{L}]}\end{array}$ & $295.17 \pm 11.69$ & $329.58 \pm 14.35^{b, d, e}$ & $318.17 \pm 13.52^{b, c}$ & $311.92 \pm 12.7^{a}$ & $301.58 \pm 10.87$ \\
\hline Serum osteocalcin [ng/mL] & $3.54 \pm 0.04$ & $1.04 \pm 0.05^{b, d, f, g}$ & $2.64 \pm 0.06 \mathrm{~b}, \mathrm{~d}, \mathrm{f}$ & $2.96 \pm 0.07^{b, d}$ & $3.23 \pm 0.07^{b}$ \\
\hline
\end{tabular}

Results are expressed as mean \pm standard deviation, $n=6$. Significance of differences between groups was evaluated by one-way ANOVA followed by Bonferroni post-hoc test; $a-p<0.05$ vs. control group; $b-p<0.0001$ vs. control group; $c-p<0.05$ vs. diabetes/insulin/PTH group; $d-p<0.0001$ vs. diabetes/insulin/PTH group; $e-p<0.05$ vs. diabetes/ /PTH group; $f-p<0.0001$ vs. diabetes/PTH group; $g-p<0.0001$ vs. diabetes/insulin group

Table 4. Morphometric parameters

\begin{tabular}{lccccc}
\hline Femur bone & Control & Diabetes & Diabetes/insulin & Diabetes/PTH & $\begin{array}{c}\text { Diabetes/insulin/ } \\
\text { /PTH }\end{array}$ \\
\hline Shaft diameter $[\mathrm{mm}]$ & $4.25 \pm 0.16$ & $3.87 \pm 0.14^{\mathrm{b}, \mathrm{c}}$ & $3.97 \pm 0.14^{\mathrm{b}}$ & $4.03 \pm 0.15^{\mathrm{a}}$ & $4.11 \pm 0.15$ \\
Neck diameter [mm] & $3.60 \pm 0.14$ & $3.19 \pm 0.13^{\mathrm{b}, \mathrm{d}, \mathrm{e}}$ & $3.29 \pm 0.13^{\mathrm{b}}$ & $3.37 \pm 0.13^{\mathrm{a}}$ & $3.45 \pm 0.14$ \\
Shaft length [mm] & $36.50 \pm 1.69$ & $35.45 \pm 1.36$ & $35.85 \pm 1.26$ & $36.06 \pm 1.35$ & $36.31 \pm 1.42$ \\
Femur weight [mg] & $502.08 \pm 17.62$ & $440.67 \pm 13.44^{\mathrm{b}, \mathrm{d}, \mathrm{e}}$ & $455.17 \pm 13.52^{\mathrm{b}}$ & $461.18 \pm 13.71^{\mathrm{b}}$ & $490.09 \pm 16.68$ \\
\hline
\end{tabular}

Results are expressed as mean \pm standard deviation, $n=12$. Significance of differences between groups was evaluated by one-way ANOVA followed by Bonferroni post-hoc test; $a-p<0.01$ vs. control group; $b-p<0.0001$ vs. control group; $c-p<0.01$ vs. diabetes/insulin/PTH group; $d-p<0.0001$ vs. diabetes/insulin/PTH group; $e-p<0.05$ vs. diabetes/ /PTH group; PTH — parathyroid hormone

with control, diabetes/PTH, and diabetes/insulin/PTH groups. Rats from diabetes/PTH group showed a statistically significant increase in alkaline phosphate when compared with controls. Also, rats from diabetes/insulin group showed a statistically significant increase in alkaline phosphate when compared with both control and diabetes/insulin/PTH groups (Table 3).

Regarding the level of serum osteocalcin, it was observed that this level was decreased to a large extent in untreated rats in diabetes group to be only $29.4 \%$ of that in control group. This decrease was also observed to a less extent in the other groups to be $74.6 \%, 83.6 \%$, and $91.2 \%$ in diabetes/insulin, diabetes/PTH, and diabetes/insulin/ PTH groups, respectively, of that in control group. When compared with control group, there was a statistically significant decrease in the level of osteocalcin in the other four groups. Rats in diabetes, diabetes/insulin, and diabetes/PTH groups showed a statistically significant decrease when compared with diabetes/insulin/PTH group. Also, there was a statistically significant decrease in both diabetes and diabetes/insulin groups when compared with diabetes/PTH group, and in diabetes/insulin group when compared with diabetes/PTH group (Table 3 ).

\section{Morphometric parameters}

Morphometric analysis was carried out through measuring shaft diameter, neck diameter, shaft length and dry weight of the femur bone. The rats in diabetes group revealed the lowest measurements for shaft diameter and neck diameter, with a statistically significant decrease in the shaft diameter when compared with both control and diabetes/insulin/ PTH groups. At the same time, there was a statistically significant decrease in the neck diameter when compared with control, diabetes/PTH, and diabetes/ insulin/PTH groups. Rats from both diabetes/insulin and diabetes/PTH groups showed a statistically significant decrease in the diameter of both shaft and neck of femur bone. Regarding the length of femur bone shaft, the length varied, but there were no significant differences between all study groups (Table 4).

The femur weight was reduced in diabetes/insulin/PTH, diabetes/PTH, diabetes/insulin and diabetes groups. There was a statistically significant decrease in the femur weight in the diabetes group when compared with control, diabetes/PTH, and diabetes/ insulin/PTH groups. Also, there was a statistically significant decrease in the femur weight in both diabetes/insulin and diabetes/PTH groups when compared with control group (Table 4).

\section{Histological results}

Microscopic examination of the bone sections from control groups (Fig. 1) showed normal bone architecture of outer cortical bone and inner spongy bone. The cortical bone was covered by periosteum 

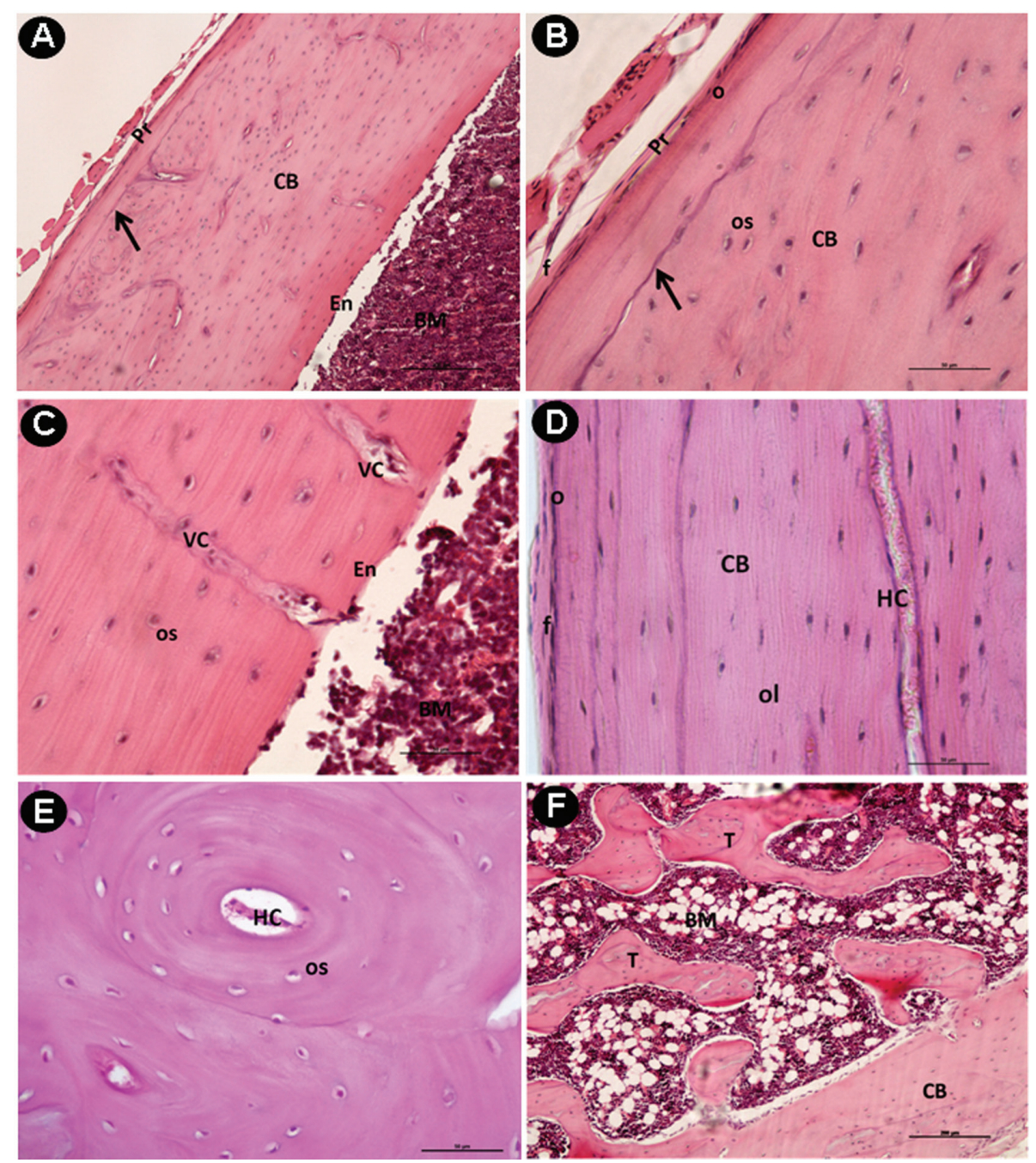

Figure 1. A-F. Photomicrographs of different sections of the distal femur diaphysis from control rats showing outer cortical bone (CB) with normal cavity appearance and osteocytes (Os) inside their lacunae. The bone is covered from outside by the periosteum (Pr), which shows both an outer fibrous layer (f) and an inner osteogenic layer (0), and from inside by smooth endosteal surface (En). Notice subperiosteal bone deposition appearing as distinct basophilic cement line (arrow). The cortical bone tissue is well organised, showing outer circumferential lamellae (ol) arranged around the Haversian canals (HC). Also, Volkmann's canals (VC) are observed. Spongy bone is composed of a network of bony trabeculae (T) separated by interconnecting spaces containing bone marrow (BM); haematoxylin and eosin; $\times 100: A, F ; \times 400: B, C, D ; \times 600: E$.

from outside and by endosteum from inside. The periosteum was formed of outer fibrous layer and inner osteogenic layer of densely packed osteoprogenitor and osteoblast cells. The cortical bone showed subperiosteal bone deposition appearing as a distinct basophilic area demarcating the border between newly added bone matrix and the older bone (Fig. 1A, B). The endosteal surface of the cortical bone appeared smooth and was lined with osteoprogenitor and osteoblastic cells with osteoclasts residing in their Howship's lacunae (Fig. 1C). The cortical bone tissue was formed of outer circumferential lamellae, concentric lamellae around Haversian canals and in- terstitial lamellae in between them. Osteocytes with their darkly stained oval nuclei residing inside their lacunae were seen in and between different lamellae (Fig. 1D). Higher magnification showed normal sized Haversian canals and regular arrangement of the lamellae of the matrix (Fig. 1E). The inner spongy bone consisted of a network of bony trabeculae separated by interconnecting spaces containing bone marrow containing developing marrow cells, blood vessels and few fat cells. The bone trabeculae consisted of irregular bone lamellae of basophilic stainability and osteocytes within their lacunae in between bone lamellae (Fig. 1F). 

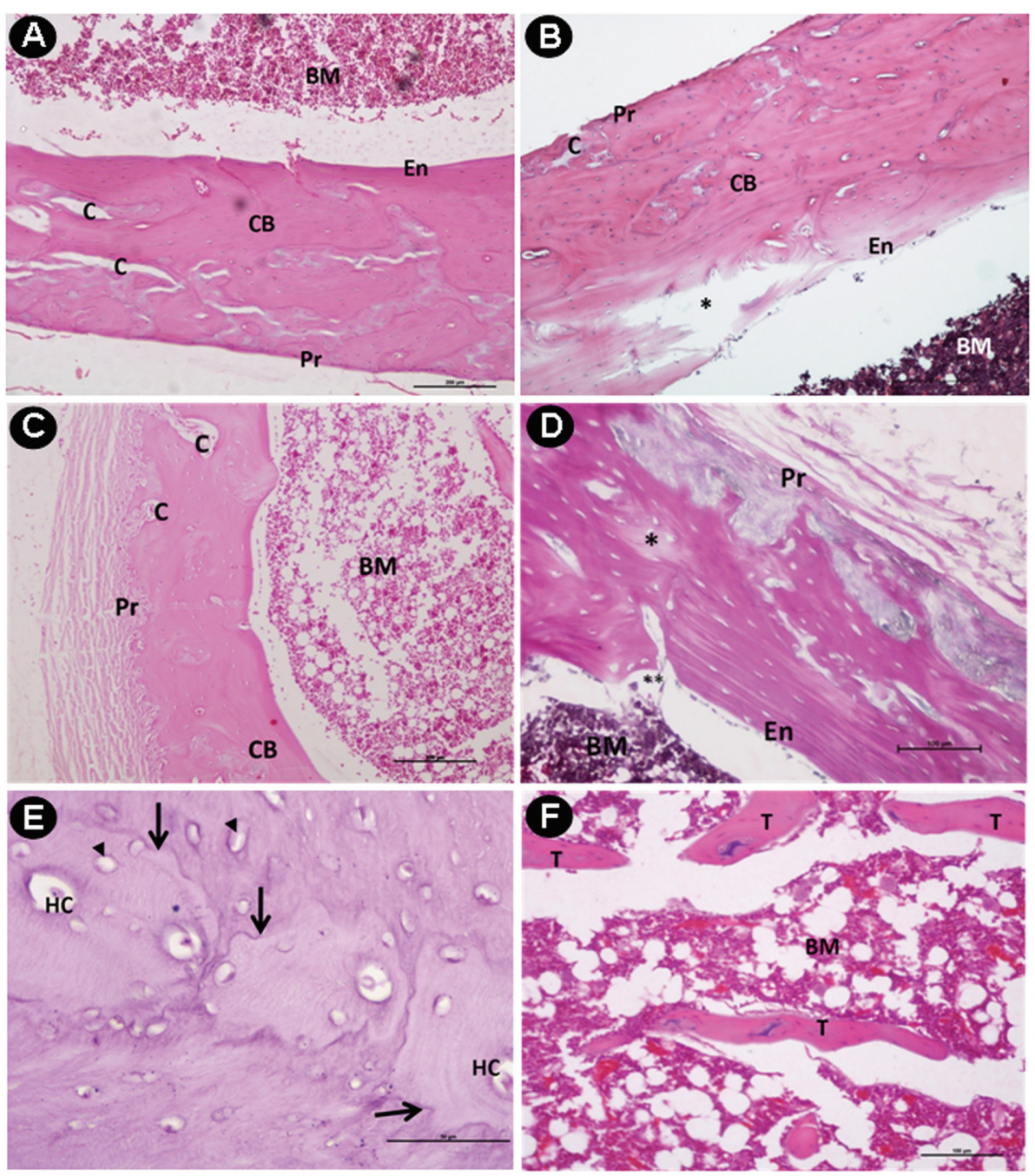

Figure 2. A-F. Photomicrographs of different sections of the distal femur diaphysis from diabetic rats showing thinning of the cortical bone (CB) with no signs of subperiosteal new bone deposition, multiple cavity formation (C), resorption cavities $\left({ }^{*}\right)$. Notice thick and irregular periosteum (Pr), irregular endosteal surface (En) with multiple eroded areas $\left({ }^{* *}\right)$. Irregular Haversian systems with irregular lamellae (arrow) and many empty lacunae (arrowhead) around Haversian canal $(\mathrm{HC})$. Spongy bone shows decreased number, size, and density of trabeculae (T); $\mathrm{BM}$ - bone marrow; haematoxylin and eosin; $\times 100: \mathrm{A}, \mathrm{C} ; \times 200: \mathrm{B}, \mathrm{F} ; \times 400: \mathrm{D} ; \times 600$ : $\mathrm{E}$.

In diabetic rats, the microscopic examination showed many histopathological changes when compared with that of control group. In some sections, thinning of the cortical bone with presence of bony grooves and tunnels and formation of multiple cavities were seen (Fig. 2A, B). In other sections, there was apparent hypertrophy of both layers of periosteum with flattened osteoprogenitor and rounded osteoblasts also forming grooves and tunnels inside compact bone (Fig. 2C). The endosteum had irregular surface with multiple notches and showed an increase in number of osteoclasts and decreased lining osteoblasts (Fig. 2D). The Haversian canals appeared shrunken with irregular outlines and forming gaps within the bone tissue. Most of osteocytes ap- peared degenerated with pyknotic nuclei. There were some empty lacunae devoid of cells and multiple osteoporotic cavities of different sizes (Fig. 2E). The inner spongy bone trabeculae showed loss of their normal architecture with widening of the interconnecting bone marrow spaces. Cavities also appeared in bone trabeculae near the medullary cavity, which was mostly occupied by fat cells (Fig. 2F).

In the diabetic rats treated with insulin alone, the examination showed slight improvement in osteoporotic changes. There was a relative increase in the thickness of cortical bone with some small osteoporotic cavities and some cement lines indicating bone repair. Outer and inner bone surfaces appeared slightly irregular (Fig. 3A, B). Bone matrix 


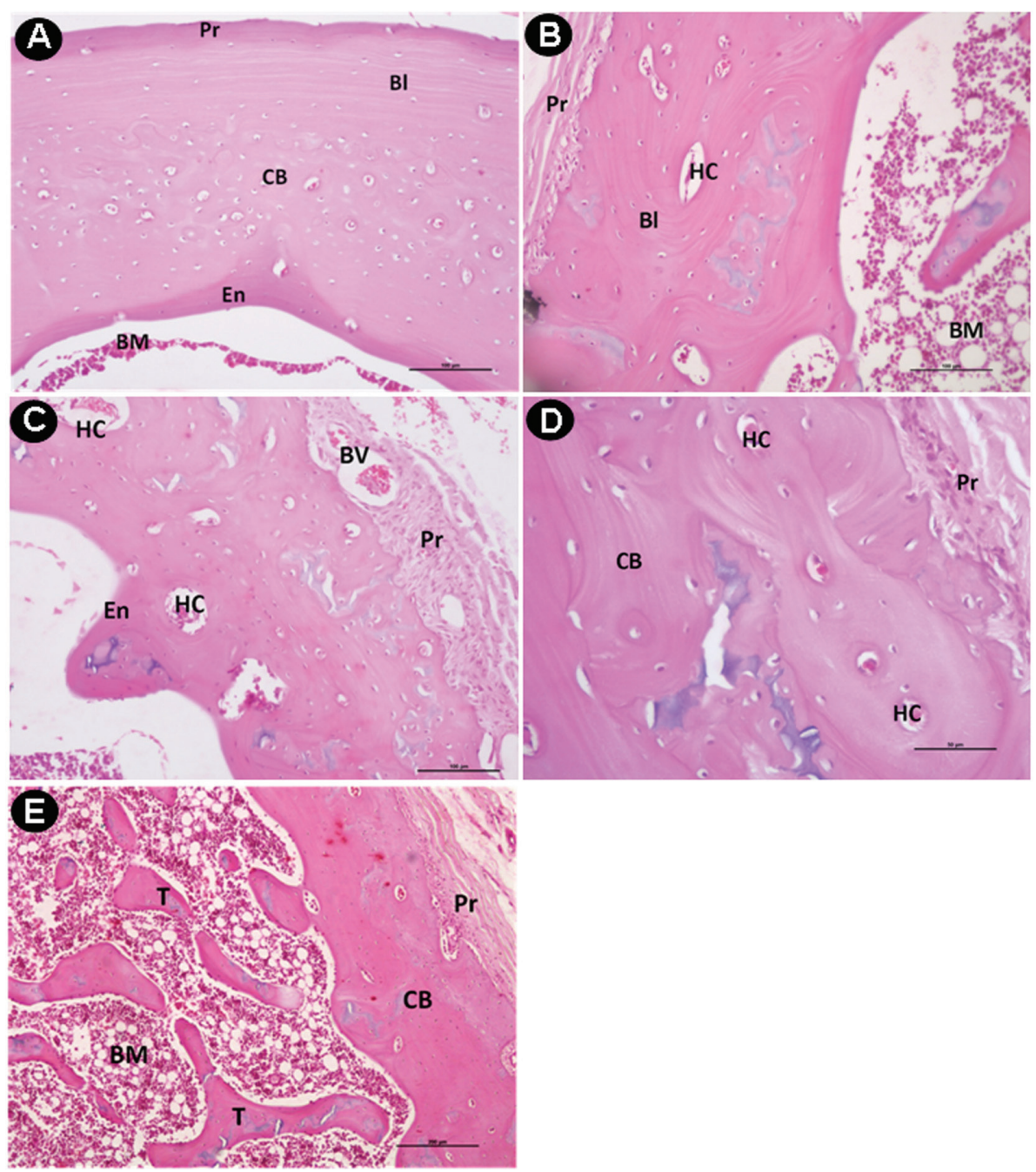

Figure 3. A-E. Photomicrographs of different sections of the distal femur diaphysis from diabetic rats treated with insulin showing the cortical bone (CB) relatively increased in thickness to be comparable with the controls with the appearance of many small cavities. The outer and inner bone surfaces appeared slightly irregular. Notice the regularly arranged bone lamellae (BI), underneath the periosteum $(\operatorname{Pr})$ and around apparently normal Haversian canals (HC). Both layers of periosteum were increased with the appearance of many blood vessels (BV). Spongy bone appears with nearly normal trabeculae (T) with less apparent widening in the interconnecting bone marrow spaces; $\mathrm{BM}$ - bone marrow; En - endosteum; haematoxylin and eosin; $\times 100: \mathrm{E} ; \times 200: \mathrm{A}, \mathrm{B}, \mathrm{C} ; \times 600$ : D.

presented areas of deep stainability starting from the endosteal bone surface. There were dilated cavities with central vascular core. These cavities were seen to be surrounded by clear deeply stained cement lines (Fig. 3C). The Haversian systems showed irregular organisation, with irregular concentric bone lamellae around apparently normal Haversian canals (Fig. 3D). Moreover, the inner spongy bone presented slight widening in the interconnecting bone marrow spaces, which contained scattered fat cells. The spongy bone trabeculae presented distinct deep basophilic cement lines and areas in the core of the trabeculae (Fig. 3E).

In the diabetic rats treated with PTH alone, the examination showed better improvement of osteoporotic changes. There was an increase in the thick- ness of cortical bone with few small osteoporotic cavities and some distinct basophilic cement lines indicating bone repair. Outer and inner bone surfaces appeared more or less regular (Fig. 4A, B). The cortical bone contained osteocytes within their lacunae and many blood vessels (Fig. 4C). Higher magnification displayed adjacent Haversian systems, which were normal in size and shape and with normally appearing concentric lamellae and osteocytes (Fig. 4D). In addition, in the inner spongy bone, many branching and anastomosing bony trabeculae containing osteocytes in their lacunae were observed arising from the cortical bone (Fig. 4E).

In the diabetic rats treated with both insulin and PTH, the examination showed a nearly complete re- 

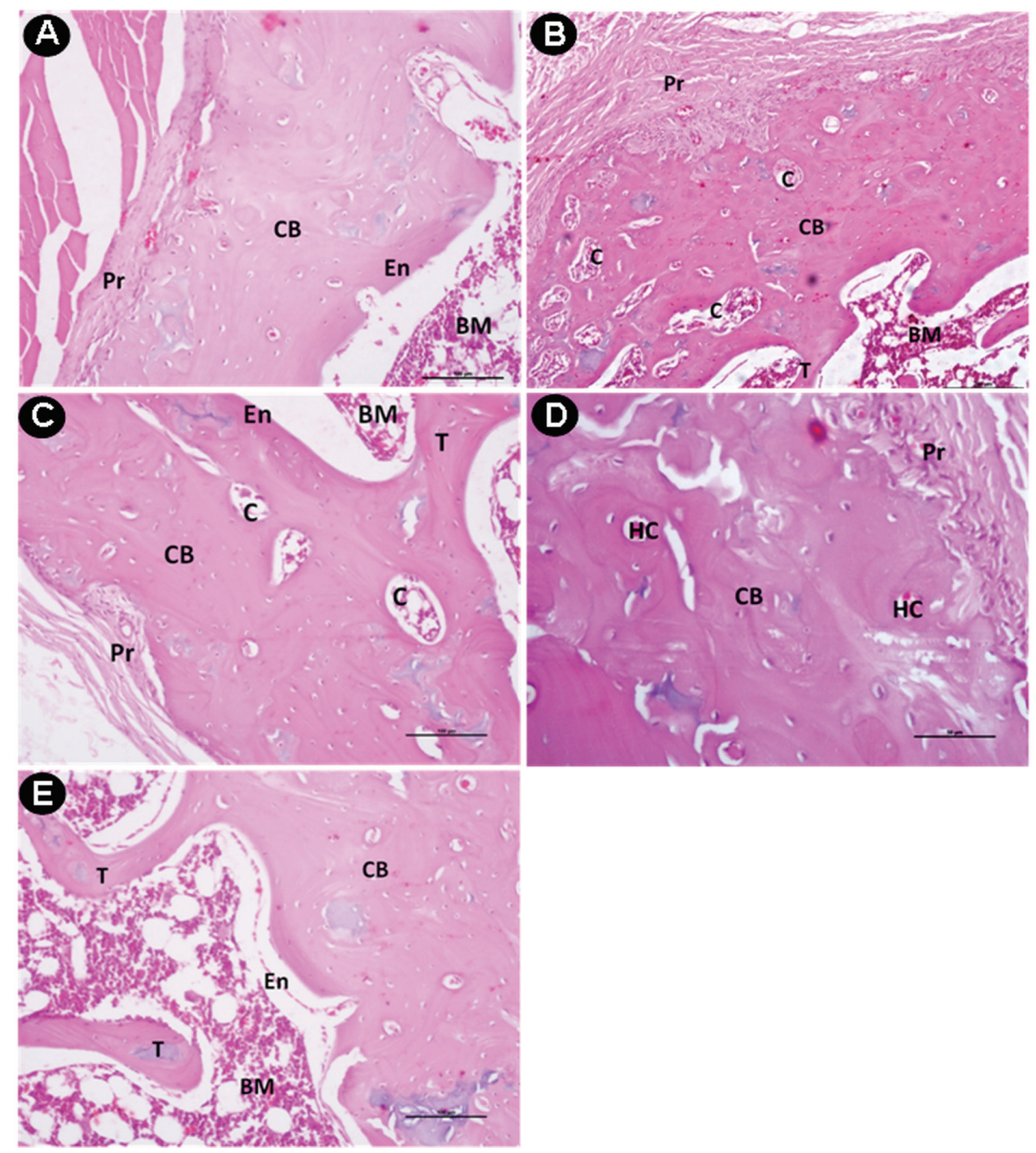

Figure 4. A-E. Photomicrographs of different sections of the distal femur diaphysis from diabetic rats treated with parathyroid hormone showing relative thickening of the cortical bone (CB) with less bony grooves and more cavities (C). The Haversian systems ( $\mathrm{HC}$ ) are normal in size and shape and with normally appearing concentric lamellae. Spongy bone appears with nearly normal trabeculae (T) with less apparent widening in the interconnecting bone marrow spaces; $\mathrm{Pr}$ - periosteum; En — endosteum; BM — bone marrow; haematoxylin and eosin; $\times 200: \mathrm{A}, \mathrm{B}, \mathrm{C}, \mathrm{E} ; \times 600$ : D.

versibility of the osteoporotic changes, where there was increased thickness of the outer cortical bone to be comparable to the control femurs with the appearance of less bony tunnels containing osteoprogenitor cells and osteoblast. Also, subperiosteal bone deposition showing a distinct basophilic cement line was seen (Fig. 5A, B). Also, there was a relative increase in the thickness of the inner osteogenic layer of the periosteum with the appearance of many rounded nuclei of osteoblasts. The endosteum had smooth surface and was lined with osteoprogenitor cells and osteoblasts (Fig. 5C). The cortical bone showed concentric bone lamellae around apparently normal Haversian canals (Fig. 5D). The inner spongy bone was resuming nearly normal thickness and architecture with less apparent widening in the interconnecting bone marrow spaces. The bone trabeculae presented distinct deep basophilic cement lines and areas in the core of the trabeculae (Fig. 5E).

\section{DISCUSSION}

The relationship between DM and osteoporosis is complex. DM causes osteopoenia and hinders fracture healing [42]. The impaired bone formation was evidenced by a decrease in both BMD and markers of bone formation as serum levels of osteocalcin and alkaline phosphatase $[8,21]$. Several mechanisms have been proposed: changes in cell signalling, in circulating growth factors and in endocrine hormones; an increase in the inflammatory process, oxidative stress or cell death [37]. It was suggested that during growth DM can lead to decrease in bone forma- 

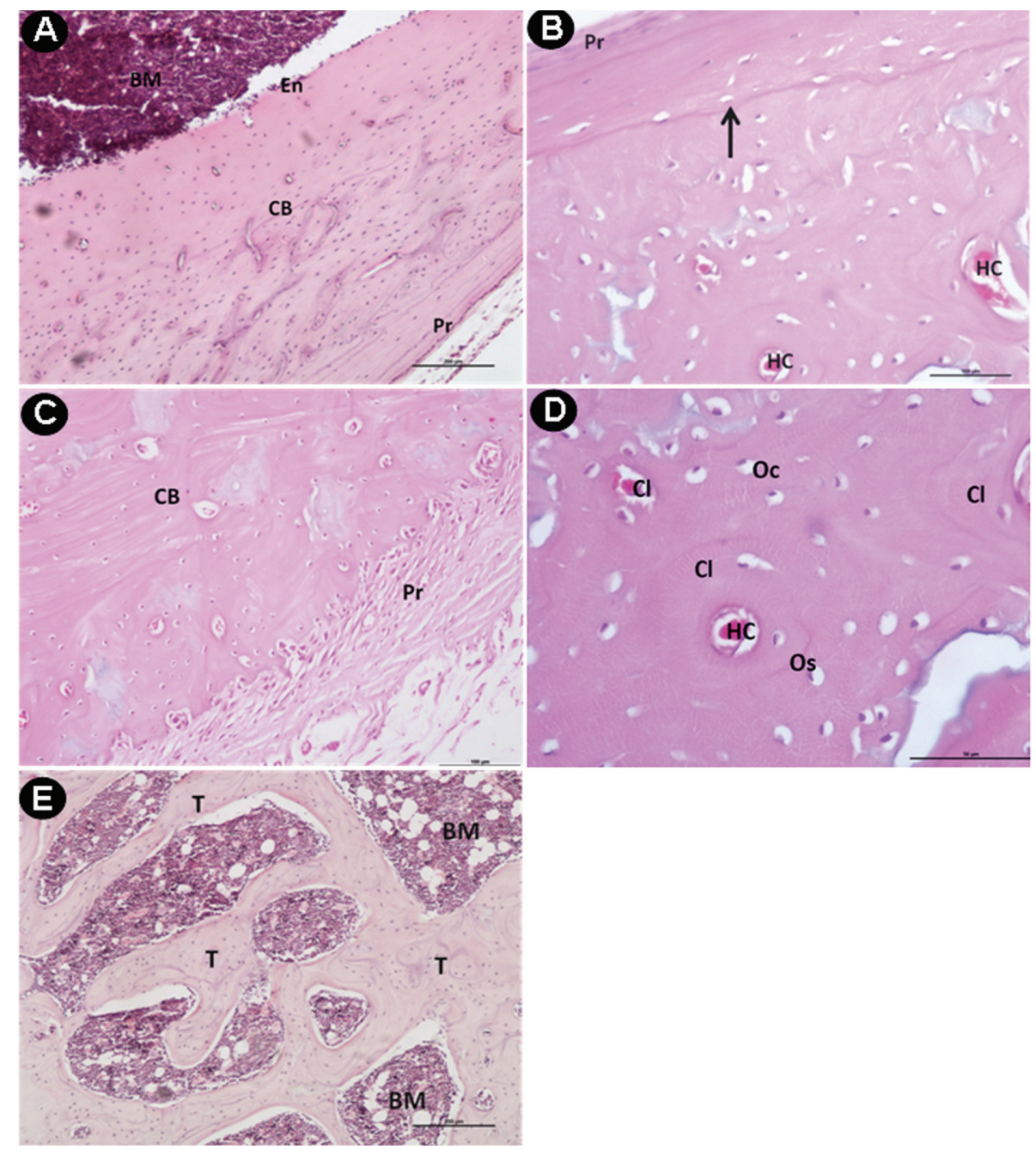

Figure 5. A-E. Photomicrographs of different sections of the distal femur diaphysis from diabetic rats treated with both insulin and parathyroid hormone showing increased thickness of the outer cortical bone (CB) to be comparable with the controls with the appearance of subperiosteal bone deposition with a distinct basophilic cement line (arrow). The cortical bone (CB) showed concentric bone lamellae (CI) around apparently normal Haversian canals $(\mathrm{HC})$. Notice the presence of many osteocytes $(\mathrm{Os})$ inside their lacunae. The periosteum $(\mathrm{Pr})$ shows increase in the thickness of its outer fibrous and inner osteogenic layers. The spongy bone appears with normal trabeculae (T) with less apparent widening in the interconnecting bone marrow spaces. The trabeculae presented with distinct deep basophilic cement lines and areas; $\mathrm{BM}$ - bone marrow; En - endosteum; haematoxylin and eosin; $\times 100: A, E ; \times 200: B, C ; \times 600$ : D.

tion, while later in life it leads to an increase in bone resorption and osteopoenia. It is thus confirmed in many studies that DM can contribute to osteopoenia by increasing osteoclast formation [52].

In the present study, following induction of diabetes, animals displayed the expected symptoms of insulin-dependent DM, i.e., hyperglycaemia, polydipsia, depression of body mass gain, and the increase in food and water intake. Administration of insulin to diabetic rats caused a reduction in food and water intake and an increase in the body weight gain. These data were in agreement with those reported by Havel et al. [20] and Akbarzadeh et al. [1].
In the present study, the diabetic rats revealed a decreased BMD and several bone changes which indicated the process of osteoporosis. The existing relation between $B M D$, the metabolism in patients with DM and osteoporosis is still in controversy. Some studies revealed no significant difference in BMD values at the hip region between diabetic and normal control women [49]. Others reported low bone density in type 2 diabetic patients [34].

The biochemical results in this study showed that diabetic rats displayed a highly significant increase in the levels of serum alkaline phosphatase and osteocalcin. Alkaline phosphatase is an enzyme marker 
of osteoblasts. It was used as a marker for bone formation due to its role in bone mineralisation and matrix production [58]. The impaired differentiation and maturation of osteoblasts in DM resulting in decreased bone formation can be traced by the decrease of serum osteocalcin levels as well as reduced mRNA levels of osteocalcin [8, 17].

In the present study, the untreated diabetic rats have displayed a decreased femoral length, weight and cortical thickness. Several studies examining physical properties of diabetic bone showed that hindrance of bone growth and healing is associated with a decreased mechanical strength of bone and not in bone production [18]. Interestingly, Reddy et al. [43] found that the strength-related properties are reduced in DM. These changes might be related to possible injurious effects of DM on bone mineralisation. However, it has been agreed that that mechanical and physical properties are not affected by DM. Moreover, insulin treatment can improve bone breaking strengths, which suggests that deficiency of bone is an effect associated with DM [23].

The diabetic rats in the present study, revealed marked thinning of the cortical bone and bone trabeculae with their wide separation and areas of osteolysis, which appeared as faint staining with absence of osteocyte lacunae. Also, multiple erosion cavities were frequently seen in the endosteal surface of cortical bone and spongy bone in the diabetic rats. It was reported that osteoporosis is manifested in the trabeculae of spongy bone as thinning, total removal or combination of thinning and total loss. This might be associated with residual trabeculae of normal thickness [50]. Similarly, Devesh et al. [13] reported that the histopathology results of diabetic rats showed destruction of medullary cavity at the expense of the bone tissue thickness and gaps formation in Haversian canals within the bone tissue.

In the present study, degeneration of osteoblasts and osteocytes, lacunae devoid of cells, multiple erosion cavities; decreased collagen fibres and irregular bone surface were observed. In accordance with these observations, it was reported that bone turnover was depressed as evidenced by a decrease in the number of osteoblasts and in osteocalcin synthesis as well as inhibition of osteoblastic activities and proliferation [32]. Inhibition of osteoblast might be due to absence of the anabolic effects of insulin [22], decreased production of growth fac- tors that regulate osteoblast differentiation [21], and apoptosis of osteoblast [44]. Moreover, osteopoenia in DM could result from hindering of bone matrix [45]. Also, it was reported that insulin plays a significant role in bone health in DM [54].

In the present study, insulin treatment of the diabetic rats resulted in relative improvement in BMD, biochemical and morphometric parameters as well as the bone osteoporotic changes, in accordance with these observations, it was reported that controlled insulin therapy may reverse the impairment in fracture repair in diabetic patients with poor metabolic control [7]. Also, localised insulin therapy improved fracture healing in diabetic animal models via chondrogenesis and cellular proliferation [19].

The results of the present study demonstrated more favourable effect of PTH treatment of diabetic rats on the BMD, biochemical and morphometric results as well as the histological appearance of bone tissue. Similarly, it was reported that PTH therapy of STZ-diabetic rats also enhances bone formation markers and bone density [34]. Furthermore, periosteal activity was noticed as indicated by the apparently increased thickness of the inner osteogenic layer of the periosteum, which contained abundant osteoblasts. Many investigators found abundant basophilic osteoblasts in the active periosteum [62]. Also, it was mentioned that periosteal bone formation could be a compensatory mechanism to keep bone strength in case of bone loss resulting from trabecular and endocortical surfaces [12].

The mechanism of PTH action in bone depends on its dose. The dose of PTH $(6.0 \mu \mathrm{g} / \mathrm{kg} /$ day $)$ in the present study was more clinically relevant compared with previous studies using higher doses in animal models $[14,36]$. Chronic elevation of serum PTH level causes net bone resorption and calcium release [29]. However, intermittent PTH treatment causes a net increase in bone formation and reduction of fracture risk [5]. Several previous studies have shown that insulin-like growth factor 1 modulates the anabolic effects of PTH on osteoblasts or bone [38]. These findings suggest that the PTH signalling pathway may interact with an insulin-like growth factor 1 signalling pathway at one or more points.

In the present study, the combined treatment with both insulin and PTH of the experimentally induced diabetic rats has nearly reversed the 
osteoporotic changes to the normal architecture and produced best results regarding morphometric, biochemical and BMD in comparison with the control rats. Moreover, the histological evaluation showed that combined insulin and PTH treatment had a greater anabolic effect and retained almost normal structure of the cortical and spongy bones than treatment with PTH or insulin alone by increasing the bone formation considerably. Although PTH and insulin are expected to have anabolic effects on diabetic rats, the present study demonstrated that PTH or insulin alone is less effective than a combination of these agents.

Because of PTH therapeutic ability to restore bone density to normal levels after bone loss, it might be an option to promote the depressed bone formation and resorption in diabetic patients. The long-term safety and mechanisms of PTH action on diseased bone and its repair is a future question that needs to be addressed in clinical studies [40].

\section{CONCLUSIONS}

It was clearly observed that untreated DM in diabetic group resulted in a dramatic reduction in BMD, morphometric and biochemical parameters. Treatment with insulin ameliorated these effects to some extent, while PTH co-treatment had a more positive effect than insulin. The combination of PTH and insulin as a treatment of DM resulted in more improvement of all parameters to approximately like that of control rats, which mean that PTH had a synergetic effect with insulin in the amelioration of the osteoporotic effects of experimental DM.

\section{REFERENCES}

1. Akbarzadeh A, Norouzian D, Mehrabi MR, Jamshidi Sh, Farhangi A, Allah Verdi A, Mofidian SMA, Lame Rad B (2007) Induction of diabetes by streptozotocin in rats. Indian J Clin Bioch, 22: 60-64.

2. Aleksynienè R, Hvid I (2004) Parathyroid hormone: possible future drug for orthopedic surgery. Medicina (Kaunas, Lithuania), 40: 842-849.

3. American Diabetes Association (2009) Diagnosis and classification of diabetes mellitus. Diabetes Care, 32: 62-67.

4. Andreassen TT, Ejersted C, Oxlund H (1999) Intermittent parathyroid hormone (1-34) treatment increases callus formation and mechanical strength of healing rat fractures. J Bone Miner Res, 14: 960-968.

5. Barnes GL, Kakar S, Vora S, Morgan EF, Gerstenfeld LC, Einhorn TA (2008) Stimulation of fracture-healing with systemic intermittent parathyroid hormone treatment. J Bone Joint Surg Am, 90: 120-127.
6. Bayat M, Abdi S, Javadieh F, Mohsenifar Z, Rashid MR (2009) The effects of low-level laser therapy on bone in diabetic and no diabetic rats. Photomed Laser Surg, 27: 703-708.

7. Beam H, Parsons J, Lin S (2002) The effects of blood glucose control upon fracture healing in the BB Wistar rat with diabetes mellitus. J Orthop Res, 20: 1210-1216.

8. Botolin S, McCabe LR (2007) Bone loss and increased bone adiposity in spontaneous and pharmacologically induced diabetic mice. Endocrinology, 148: 198-205.

9. Brown SA, Sharpless JL (2004) Osteoporosis: an under-appreciated complication of diabetes. Clinical Diabetes, 22: 10-20.

10. Chau DL, Edelman SV (2002) Osteoporosis and diabetes. Clin Diabetes, 20: 153-157.

11. Chau DL, Edelman SV, Chandran M (2003) Osteoporosis and diabetes. Curr Diabet Rep, 3: 37-42.

12. Chen $H$, Tian $X$, Liu $X$, Setterberg RB, Li M, Jee WSS (2008) Alfa calcidol-stimulated focal bone formation on the cancellous surface and increased bone formation on the periosteal surface of the lumbar vertebrae of adult female rats. Calcif Tissue Int, 82: 127-136.

13. Devesh C, Kritika M, Anroop N, Kumar SP, Sumeet G (2012) Spirulina reverses histomorphological changes in diabetic osteoporosis in Pioglitazone treated rats. J Diabetes Metab, S1: 1-7.

14. Dobnig H, Turner RT (1997) The effects of programmed administration of human parathyroid hormone fragment (I-34) on bone histomorphometry and serum chemistry in rats. Endocrinology, 138: 4607-4612.

15. Follak N, Kloting I, Wolf E, Merk H (2004a) Histomorphometric evaluation of the influence of the diabetic metabolic state on bone defect healing depending on the defect size in spontaneously diabetic BB/OK rats. Bone, 35: 144-152.

16. Follak N, Kloting I, Wolf E, Merk H (2004b) Improving metabolic control reverses the histomorphometric and biomechanical abnormalities of an experimentally induced bone defect in spontaneously diabetic rats. Calcif Tissue Int, 74: 551-560.

17. Fowlkes JL, Bunn RC, Liu L,Wahl EC, Coleman HN, Cockrell GE, Perrien DS, Lumpkin CK Jr, Thrailkill KM (2008) Runt-related transcription factor 2 (RUNX2) and RUNX2-related osteogenic genes are down-regulated throughout osteogenesis in type 1 diabetes mellitus. Endocrinology, 149: 1697-1704.

18. Funk JR, Hale JE, Carmines D, Gooch HL, Hurwitz SR (2000) Biomechanical evaluation of early fracture healing in normal and diabetic rats. J OrthopedicRes, 18: 126-132.

19. Gandhi A, Beam HA, O'Connor JP, Parsons JR, Lin SS (2005) The effects of local insulin delivery on diabetic fracture healing. Bone, 37: 482-490.

20. Havel PJ, Hahn TM, Sindelar DK, Baskin DG, Dallman MF, Weigle DS, Schwartz MW (2000) Effects of streptozotocin-induced diabetes and insulin treatment on the hypothalamic melanocortin system and muscle uncoupling protein 3 expression in rats. Diabetes, 49: 243-252.

21. He H, Liu R, Desta T, Leone C, Gerstenfeld LC, Graves DT (2004) Diabetes causes decreased osteoclastogenesis, 
reduced bone formation and enhanced apoptosis of osteoblastic cells in bacteria stimulated bone loss. Endocrinology, 145: 447-452.

22. Hofbauer LC, Brueck CC, Singh SK, Dobnig H (2007) Osteoporosis in patients with diabetes mellitus. J Bone Mineral Res, 22: 1317-1318.

23. Holzhausen M, Garcia DF, Pepato MT, Marcantonio E (2004) The influence of short-term diabetes mellitus and insulin therapy on alveolar bone loss in rats. J Periodont Res, 39: 188-193.

24. Ikeda T, Manabe H, Iwata K (2004) Clinical significance of alendronate in postmenopausal type 2 diabetes mellitus. Diabetes Metab, 30: 355-358.

25. Inzerillo AM, Epstein S (2004) Osteoporosis and diabetes mellitus. Rev Endocr Metab Disord, 5: 261-268.

26. 26. Jackuliak P, Payer J (2014) Osteoporosis, fractures, and diabetes: review article. Int J Endocrinol, 2014: 820615.

27. Jahng JS, Kim HW (2000) Effect of intermittent administration of parathyroid hormone on fracture hea1ing in ovariectomized rats. Orthopedics, 23: 1089-1094.

28. Janghorbani M, Van Dam RM, Willett WC, Hu FB (2007) Systematic review of type 1 and type 2 diabetes mellitus and risk of fracture. Am J Epidemiol, 166: 495-505.

29. Jilka RL, O'Brien CA, Bartell SM, Weinstein RS, Manolagas SC (2010) Continuous elevation of PTH increases the number of osteoblasts via both osteoclast-dependent and independent mechanisms. J Bone Miner Res, 25: 2427-2437.

30. Kasukawa $Y$, Miyakoshi N, Itoi E, Tsuchida T, Tamura $Y$, Kudo T, Suzuki K, Seki A, Sato K (2004) Effects of h-PTH on cancellous bone mass, connectivity and bone strength in ovariectomized rats with and without sciatic neurectomy. J Orthop Res, 22: 457-464.

31. Khamaisi M, Regev E, Yarom N, Avni B, Leitersdorf E, Raz I, Elad S (2007) Possible association between diabetes and bisphosphonate-related jaw osteonecrosis. J Clin Endocrinol Metab, 92: 1172-1175.

32. Liu Z, Aronson J, Wahl EC, Liu L, Perrien DS, Kern PA, Fowlkes JL, Thrailkill KM, Bunn RC, Cockrell GE, Skinner RA, Lumpkin CK Jr (2007) A novel rat model for the study of deficits in bone formation in type-2 diabetes. Acta Orthopaedica, 78: 46-55.

33. Lopez Ibarra PJ, Pastor MM, Escobar Jimenez F, Pardo MD, Gonzalez AG, Luna JD, Requena ME, Diosdado MA (2001) Bone mineral density at time of clinical diagnosis of adultonset type 1 diabetes mellitus. Endocr Pract, 7: 346-351.

34. Lozano D, De Castro LF, Dapia S, Andrade Zapata I, Manzarbeitia F, Alvarez Arroyo MV, Gómez-Barrena E, Esbrit P (2009) Role of parathyroid hormone-related protein in the decreased osteoblast function in diabetes-related osteopenia. Endocrinology, 150: 2027-2035.

35. Makino H, Tanaka I, Mukoyama M, Sugawara A, Mori K, Muro S, Suganami T, Yahata K, Ishibashi R, Ohuchida S, Maruyama T, Narumiya S, Nakao K (2002) Prevention of diabetic nephropathy in rats by prostaglandin $\mathrm{E}$ receptor EP I-selective antagonist. Am Soc Nephrol, 13: 1757-1765.
36. Mashiba T, Burr DB, Turner CH, Sato M, Cain RL, Hock JM (2001) Effects of human parathyroid hormone (1-34), LY333334, on bone mass, remodeling, and mechanical properties of cortical bone during the first remodelling cycle in rabbits. Bone, 28: 538-547.

37. McCabe LR (2007) Understanding the pathology and mechanisms of type I diabetic bone loss. J Cell Biochem, 102: 1343-1357.

38. Miyakoshi N, Kasukawa Y, Linkhart TA, Baylink DJ, Mohan S (2001) Evidence that anabolic effects of PTH on bone require IGF-I in growing mice. Endocrinology, 142: 4349-4356.

39. Moshref SS (2007) PM 701 a highly selective anti cancerous agent against L1210 leukemic cells: In vivo clinical and histopathological study. J KAU Med Sci, 14: 85-99.

40. Motyl KJ, McCauley LK, McCabe LR (2012) Amelioration of type I diabetes-induced osteoporosis by parathyroid hormone is associated with improved osteoblast survival. J Cell Physiol, 227: 1326-1334.

41. Nakazawa T, Nakajima A, Shiomi K, Moriya H, Einhorn TA, Yamazaki M (2005) Effects of low-dose, intermittent treatment with recombinant human parathyroid hormone (1-34) on chondrogenesis in. a model of experimental fracture healing. Bone, 37: 711-719.

42. Nicodemus KK, Folsom AR (2001) Type 1 and type 2 diabetes and incident hip fractures in postmenopausal women. Diabetes Care, 24: 1192-1197.

43. Reddy GK, Stehno-Bittel L, Hamade S, Enwemeka CS (2001) The biomechanical integrity of bone in experimental diabetes. Diabetes Research and Clinical Practice, 54: 1-8.

44. Saag KG, Zanchetta JR, Devogelaer JP, Adler RA, Eastell R, See K, Krege JH, Krohn K, Warner MR (2009) Effects of teriparatide versus alendronate for treating glucocorticoid-induced osteoporosis: Thirty-six-month results of a randomized, double-blind, controlled trial. Arthritis Rheum, 60: 3346-3355.

45. Saito M, Fujii K, Mori Y, Marumo K (2006) Role of collagen enzymatic and glycation induced cross-links as a determinant of bone quality in spontaneously diabetic WBN/Kob rats. Osteoporos Int, 17: 1514-1523.

46. Santana RB, Xu L, Chase HB, Amar S, Graves DT, Trackman PC (2003) A role for advanced glycation end products in diminished bone healing in type of 47 diabetes. Diabetes, 52: 1502-1510.

47. Schwartz AV (2003) Diabetes mellitus: Does it affect bone? Calcif Tissue Int, 73: 515-519.

48. Silva MJ, Brodt MD, Lynch MA, McKenzie JA, Tanouye KM, Nyman JS, Wang X (2009) Type 1 diabetes in young rats leads to progressive trabecular bone loss, cessation of cortical bone growth and diminished whole bone strength and fatigue life. J Bone Mineral Res, 24: 1618-1627.

49. Sosa $M$, Dominguez $M$, Navarro MC, Segarra MC, Hernandez D, de Pablos P, Betancor P (1996) Bone mineral metabolism is normal in non-insulin-dependent diabetes mellitus. J Diabetes Complications, 10: 201-205. 
50. Sulayman AA (2007) Effect of water soluble soybean fiber (WSSF) on bone changes induced by ovariectomy in female albino rat: a light and scanning electron microscopic study. Egypt J Histol, 30: 221-232.

51. Suzuki K, Miyakoshi N, Tsuchida T, Kasukawa Y, Sato K, Itoi $E$ (2003) Effects of combined treatment of insulin and human parathyroid hormone (1-34) on cancellous bone mass and structure in streptozotocin-induced diabetic rats. Bone, 33: 108-114.

52. Suzuki K, Kurose T, Takizawa M, Maruyama M, Ushikawa K, Kikuyama M, Sugimoto C, Seino Y, Nagamatsu S, Ishida H (2005) Osteoclastic function is accelerated in male patients with type 2 diabetes mellitus: the preventive role of osteoclastogenesis inhibitory factor/osteoprotegerin (OCIF/OPG) on the decrease of bone mineral density. Diabetes Res Clin Pract, 68: 117-125.

53. Thrailkill KM, Liu L, Wahl EC, Bunn RC, Perrien DS, Cokrell GE, Skinner RA, Hogue WR, Carver AA, Fowlkes JL, Aronson J, Lumpkin CK Jr (2005). Bone formation is impaired in a model of type 1 diabetes. Diabetes, 54: 2875-2881.

54. Torres-Duran PV, Ferreira-Hermosillo A, Juarez-Oropeza MA (2007) Antihyper-lipedemic and antihypertensive effects of spirulina maxima in an open sample of Mexican population: a preliminary report. Lipids Health Dis, 6: 33.

55. Tsuchida T, Sato K, Miyakoshi N, Abe T, Kudo T, Tamura Y, Kasukawa Y, Suzuki K (2000) Histomorphometric evaluation of the recovering effects of human parathyroid hormone (1-34) on bone structure and turnover in streptozotocininduced diabetic rats. Calcif Tissue Int, 66: 229-233.

56. Tsuchida T, Miyakosm N, Kudo T, Tamura Y, Kasukawa $Y$, Suzuki K, Sato K (2001) Restoring effects of human parathyroid hormone (1-34) on trabecular connectivity in ovariectomized rats. Tohoku J Exp Med, 194: 213-221.

57. Turner RB, Vagula M, Devi SS (2009) Osteoporosis: An understated complication of diabetes. US Pharm, 34: 14-16.

58. Uchiyama S, Yamaguchi M (2005) Oral administration of beta-cryptoxanthin prevents bone loss in streptozotocindiabetic rats in vivo. Biol Pharm Bull, 28: 1766-1769.

59. VahleJL, Sato M, Long GG, Young JK, Francis PC, EngelhardtJA, Westmore MS, Linda Y, Nold JB (2002) Skeletal changes in rats given daily subcutaneous injections of recombinant human parathyroid hormone (1-34) for 2 years and relevance to human safety. Toxicol Pathol, 30: 312-321.

60. Wild S, Roglic G, Green A, Sicree R, King H (2004) Global prevalence of diabetes: estimates for the year 2000 and projections for 2030. Diabetes Care, 27: 1047-1053.

61. World Health Organization. Diabetes. Fact sheet No. 312. November 2008. www.who.int/mediacentre/factsheets/ fs312/en/. Accessed April 2, 2009.

62. Young B, Lowe JO, Stevens A, Heath JW (2005) Wheater's functional histology. 5th Ed. Churchill Livingstone, Philadelphia. 